\title{
Estimating completeness of foodborne disease registration in North West Morocco
}

\author{
Soumaia Hmimou ${ }^{1,4^{*}}$, Abdelmajid Soulaymani ${ }^{4}$, Nesma Nekkal $^{2}$, Naima Rhalem $^{3}$, Abdelrhani Mokhtari ${ }^{4}$, François Xavier \\ Nshimiyimana ${ }^{1}$, Rachida Soulaymani-Bencheikh ${ }^{3}$, and Hinde $\mathrm{Hami}^{1}$ \\ ${ }^{1}$ Faculty of Environmental Studies, University of Lay Adventists of Kigali, Kigali, Rwanda \\ ${ }^{2}$ Interface Center and Medical Application (CIAM), Mohammed VI Polytechnic University, Ben Guerir, Morocco \\ ${ }^{3}$ Moroccan Poison Control Center, Rabat, Morocco \\ ${ }^{4}$ Laboratory of Biology and Health, Faculty of Science, Ibn Tofail University, Kenitra, Morocco
}

\begin{abstract}
This study aims to estimate the total number of poisoning cases and completeness of foodborne disease surveillance in the region of Tanger-Tétouan-Al Hoceima in North West Morocco. This retrospective study was conducted to assess the completeness of food poisoning surveillance system in the Tanger-Tétouan-Al Hoceima region over a three-year period from January 1, 2014 to December 31, 2016. The data used for this study were obtained from two sources, including the national poisoning database of the Moroccan Poison Control Center (MPCC) and provincial hospital registries in the study area. The completeness of foodborne disease reporting was estimated using the capture-recapture method. During the study period, 235 cases of foodborne diseases were reported to the Moroccan Poison Control Center, 725 cases were notified to the provincial hospital registries and 98 cases were common to both sources. The estimated total number of poisoning cases was $1,739(95 \% \mathrm{Cl}: 1,494-1,983)$. The completeness of foodborne disease surveillance for the national database of poisoning and hospital registries was estimated to be $13.5 \%$ and $41.7 \%$, respectively. Improvement of the completeness of poisoning surveillance system is needed to estimate the true incidence of poisoning in Morocco.
\end{abstract}

Keywords: Foodborne diseases; Completeness; Morocco

\section{Introduction}

Foodborne diseases are a growing public health problem responsible for substantial morbidity and mortality worldwide [1]. However, the severity of these diseases and related mortality are not accurately reflected based on data available in developed and developing countries [2]. Gastrointestinal diseases caused approximately 2 million deaths worldwide during the year 2005 in the United States [3]. The Centers for Disease Control and Prevention (CDC) estimates that each year 3000 Americans die from diseases linked to food and 8078 cases of foodborne disease were reported in Shandong province in China between 2011 and 2016 [4, 5].

In Morocco, foodborne diseases are the 3rd leading cause of poisoning with $17.2 \%$ of all causes in 2016 [6].

This type of poisoning results from the ingestion of contaminated foods and food products and includes a broad group of illnesses caused by parasites, chemicals and pathogens which contaminate food at different stages in the food production and preparation process [7]. In 1980, Morocco set up a national system of compulsory declaration of all cases of foodborne diseases for the collection and periodic communication of data to the Moroccan Poison Control Center (MPCC).

Among the main objectives of this center is the reduction of the incidence of all poisonings including foodborne diseases. The incidence of these poisonings can be calculated by the number of cases notified, assuming a complete detection and notification of cases.

The aim of this study is to estimate the total number of poisoning cases and completeness of foodborne disease surveillance in the region of Tanger-Tetouan-Al Hoceima (North West of Morocco) during the period 2014-2016.

\section{Methods}


In this study, we included all cases of foodborne diseases recorded between 2014 and 2016 in the region of TangerTétouan-Al Hoceima in Morocco.

The data used for this study were obtained from two sources:

- Moroccan Poison Control Center (MPCC) database which records all calls received at the toxicological information unit (phone calls) and also from poisoning declaration forms received at the toxicovigilance unit;

- Hospital registries (HR).

The two data sources were compared to identify common cases and the cases present in each of the sources.

The following data were collected from the two sources: date of exposure, province, age, gender, origin, residence, type of poisoning, signs and symptoms, treatment and outcome.

\section{Capture-recapture method}

The capture-recapture method is a technique derived from ecology applied to epidemiological studies to estimate the number of cases in a population including those that escape reporting and then to assess the completeness of sources [8-13].

Six conditions need to be observed to effectively apply the capture-recapture method: all the cases identified by the sources are real cases; the cases occurred during the period and in the geographic area studied; the population studied is closed; all common cases and only common cases are identified; the sources are independent of each other and there is homogeneity in the capture of cases [14, $15]$.

Assuming the independence of the two sources, Sekar and Deming propose the following estimates $[8,16]$.

$$
N=\frac{(a+b) \times(a+c)}{a}
$$

a: number of duplicates, $\mathbf{b}$ : number of cases only present in the first source, $\mathbf{c}$ : number of cases only present in the second source.

Chapman (1951) and Seber (1970) showed that these estimators can be biased when the numbers are small and that the duplicates can be equal to zero $[16,17]$.

They proposed the following unbiased estimators:

$$
N=\frac{(a+b+1)(a+c+1)}{(a+1)}-1
$$

To calculate the completeness (C) of each of two sources, we used the following formula:

$$
C=\frac{(a+b+c)}{N} * 100
$$

During the 2014-2016 period, 235 cases of foodborne diseases were reported to the Moroccan Poison Control Center and 725 cases were notified to the provincial hospital registries in the study region, for a total of 862 cases of poisoning after elimination of 98 duplicates (Figure 1).

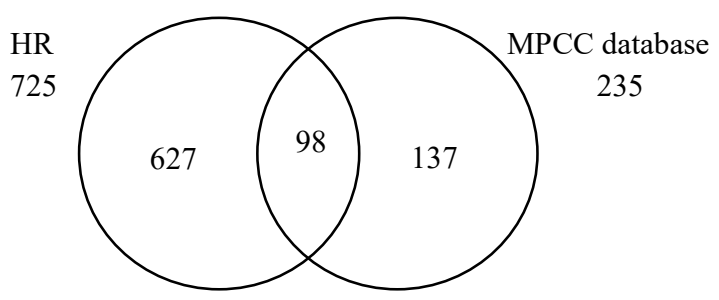

Fig. 1. Distribution of foodborne disease cases reported by two sources, Tanger-Tétouan-Al Hoceima, Morocco, 2014-2016

The average age of the patients was $24.9 \pm 14.9$ years. Most victims were teenagers and young adults aged 16-45 years $(50.72 \%)$. The male-female ratio is 1.01 . Most incidents occurred at home $(86.1 \%)$ and in urban areas $(82.90 \%)$. According to the results, the majority of poisoning cases occurred during the summer and spring months (38.40\% and 30.51\%, respectively). About 35\% of the cases were from collective poisoning.

We found that 877 cases were unreported by any source and the estimated total number of poisoning cases was 1739 (95\% IC: 1,983-1,494) (Table 1).

The completeness of foodborne disease surveillance was estimated to be $49.57 \%$ for both sources, $13.52 \%$ for the national database of poisoning and $41.70 \%$ for hospital registries in the study region.

Thus, more than half $(50.43 \%)$ of all cases were unreported by both sources.

Table 1. Intersection of two data sources, TangerTétouan-Al Hoceima, Morocco, 2014-2016

\begin{tabular}{|c|l|r|r|r|}
\cline { 3 - 5 } \multicolumn{2}{c|}{} & \multicolumn{3}{c|}{ MPCC database } \\
\cline { 3 - 5 } \multicolumn{2}{c|}{} & \multicolumn{1}{c|}{ Yes } & \multicolumn{1}{c|}{ No } & \multicolumn{1}{c|}{ Total } \\
\hline \multirow{3}{*}{$\begin{array}{c}\text { Hospital } \\
\text { registries }\end{array}$} & Yes & 98 & 627 & 235 \\
\cline { 2 - 5 } & No & 137 & 877 & - \\
\cline { 2 - 5 } & Total & 725 & - & 1739 \\
\hline
\end{tabular}

The two-source capture-recapture estimates of the number of foodborne disease cases for data stratified by year, 
season, province, age, gender, origin, type of poisoning and residence are presented in Table 2.

Based on the stratification results, the estimated total number of poisoning cases by year (1739 cases), season (1739 cases) and province (1739 cases) is equal to the overall estimate. Stratification by gender (1687 cases), type of poisoning (1552 cases) and origin (1573 cases)

Table 2. Number of cases and completeness of foodborne disease surveillance, Tanger-Tétouan-Al Hoceima, Morocco, 2014-2016 gives a lower estimate of the total number of cases but this remains within the $95 \%$ confidence interval. Also, origin (1108 cases) and age (2779 cases) lead to different estimates for the overall estimate.

\begin{tabular}{|c|c|c|c|c|c|c|}
\hline & \multicolumn{4}{|c|}{ Number of cases } & \multicolumn{2}{|c|}{ Completeness \% } \\
\hline & $\begin{array}{c}\text { Both sources } \\
(\%)\end{array}$ & Common & Estimated & Unreported & MPCC & HR \\
\hline \multicolumn{7}{|l|}{ Year } \\
\hline 2014 & $312(36.19)$ & 55 & 421 & 109 & 19.23 & 67.90 \\
\hline 2015 & $297(34.45)$ & 18 & 903 & 606 & 7.20 & 27.69 \\
\hline 2016 & $253(29.35)$ & 25 & 673 & 420 & 13.23 & 28.09 \\
\hline Total & $862(100.00)$ & 98 & 1739 & 877 & 13.52 & 41.70 \\
\hline \multicolumn{7}{|l|}{ Season } \\
\hline Autumn & $127(14.73)$ & 10 & 388 & 261 & 10.31 & 25.00 \\
\hline Winter & $141(16.36)$ & 28 & 174 & 33 & 21.21 & 75.68 \\
\hline Spring & $263(30.51)$ & 34 & 458 & 195 & 14.85 & 50.00 \\
\hline Summer & $331(38.40)$ & 26 & 924 & 593 & 9.74 & 28.89 \\
\hline Total & $862(100.00)$ & 98 & 1739 & 877 & 13.52 & 41.70 \\
\hline \multicolumn{7}{|l|}{ Province } \\
\hline Al Hoceima & $67(7.77)$ & - & 539 & 472 & 1.48 & 10.95 \\
\hline Chefchaouen & $291(33.76)$ & 40 & 635 & 344 & 19.05 & 33.06 \\
\hline Larache & $90(10.44)$ & 13 & 146 & 56 & 16.46 & 54.17 \\
\hline M'diq-Fnideq & $300(34.80)$ & 44 & 317 & 17 & 14.81 & 93.62 \\
\hline Ouezzane & $8(0.93)$ & - & 15 & 7 & 6.67 & 46.67 \\
\hline Tanger-Assilah & $78(9.05)$ & - & 1403 & 1325 & 1.78 & 3.78 \\
\hline Tétouan & $28(3.25)$ & 1 & 180 & 152 & 5.00 & 11.11 \\
\hline Total & $862(100.00)$ & 98 & 1739 & 877 & 13.52 & 41.70 \\
\hline \multicolumn{7}{|l|}{ Age group } \\
\hline Infant & $3(0.39)$ & - & 5 & 2 & 40.00 & 20.00 \\
\hline Walking baby & $43(5.62)$ & 2 & 187 & 144 & 5.88 & 18.18 \\
\hline Child & $139(18.17)$ & 9 & 422 & 283 & 7.83 & 27.27 \\
\hline Teenager & $111(14.51)$ & 2 & 966 & 855 & 2.17 & 9.52 \\
\hline Adult & $462(60.39)$ & 27 & 1544 & 1082 & 7.12 & 24.55 \\
\hline Elderly & $7(0.92)$ & - & 7 & - & 0.00 & 100.00 \\
\hline Total & $765(100.00)$ & 40 & 2779 & 2014 & 6.37 & 22.60 \\
\hline \multicolumn{7}{|l|}{ Gender } \\
\hline Female & $424(49.70)$ & 49 & 869 & 445 & 13.92 & 40.50 \\
\hline Male & $429(50.30)$ & 49 & 816 & 387 & 13.24 & 45.37 \\
\hline Total & $853(100.00)$ & 98 & 1687 & 834 & 13.57 & 42.79 \\
\hline \multicolumn{7}{|l|}{ Origin } \\
\hline Rural & $79(17.10)$ & 5 & 324 & 245 & 9.26 & 16.67 \\
\hline Urban & $383(82.90)$ & 51 & 855 & 472 & 18.48 & 32.28 \\
\hline Total & $462(100.00)$ & 56 & 1108 & 646 & 16.97 & 29.79 \\
\hline \multicolumn{7}{|c|}{ Type of poisoning } \\
\hline Collective & $273(34.73)$ & 24 & 761 & 488 & 12.50 & 27.59 \\
\hline Isolated & $513(65.27)$ & 74 & 874 & 361 & 16.16 & 50.34 \\
\hline Total & $786(100.00)$ & 98 & 1552 & 766 & 15.08 & 41.88 \\
\hline \multicolumn{7}{|l|}{ Residence } \\
\hline Home & $318(88.09)$ & 7 & 2892 & 2574 & 2.90 & 8.33 \\
\hline School & $4(1.01)$ & 1 & 6 & 2 & 50.00 & 33.33 \\
\hline Workplace & $13(3.60)$ & 6 & 15 & 2 & 75.00 & 54.55 \\
\hline Public place & $13(3.60)$ & 1 & 40 & 27 & 25.00 & 10.00 \\
\hline Restaurant & $13(3.60)$ & 5 & 13 & - & 100.00 & 38.46 \\
\hline Total & $361(100.00)$ & 20 & 1573 & 1212 & 7.69 & 16.53 \\
\hline
\end{tabular}

\section{Discussion}

Evaluating surveillance systems is not always easily conceivable because there is no way of knowing the actual epidemiological situation or the exact number of events 
that occurred. However, many studies have addressed the issue leading to the development of more precise and less biased estimators in recent decades based on a method initially applied in ecology for the census of wildlife populations: the capture-recapture method.

This method is used with two or more sources and involves six conditions. It enables the estimation of the real number of events occurring in a population during a given period through a cross analysis of events reported by several systems to identify the common events from the different systems.

In this study, we attempted to evaluate the surveillance system for foodborne diseases in the region of TangerTetouan-Al Hoceima during the period 2014-2016. The completeness of foodborne disease reporting was estimated using the two-source capture-recapture method. The two databases were compared for the identification of common cases to both sources: MPCC database and hospital registries. The variables used for comparison were: gender, age, date of exposure and province. The intersection of data from two sources led to identification of 98 common cases. It is important to know that an overestimation of common cases leads to an underestimation of the total number of cases by the capture-recapture method $[19,20]$.

The precision of estimates depends more on several conditions being respected $[21,22]$.

The first of these conditions is that all of the cases should be real cases. This condition requires a common case definition which must be respected. For this study, the definition of foodborne disease cases was all patients suffering from foodborne diseases between 2014 and 2016 in the region of Tanger-Tetouan-Al Hoceima in Morocco. Failure to comply with this condition could increase the number of false positives included and therefore lead to an overestimation of the total number of cases and an underestimation of the completeness of the sources.

The second condition is that of the study period and the geographic area which must be identical. The study concerns the foodborne disease cases recorded from January $1^{\text {st }}, 2014$ to December $31^{\text {st }}, 2016$ for both sources. The third condition which needs to be applied is that the study population should be closed. To respect this condition, there must not be a population movement so that an equiprobability of case capture in each source can be ensured. The capture probability therefore seems identical for each of the sources.

The fourth condition to be applied is that all cases in the population must have the same probability of being identified by a source which means that the catch must not be linked to certain characteristics of surveillance system or of cases.

In this study, the heterogeneity of capture was studied for 8 variables: Year, season, province, age, gender, origin, type of poisoning and residence.

We took into account the heterogeneity of the catch for the year, season, province, gender, type of poisoning and residence. This shows that the estimates fall within the confidence interval of the overall estimate.

The last application condition is that the two sources must be independent. The results show that there is no dependence between MPCC database and hospital registries. The two data sources were considered to be independent. Negative dependence was very unlikely although positive dependence (identification of a case by one source increases the probability of that case being identified by the other) was possible.

In applying the capture-recapture method, the completeness of poisoning surveillance was estimated to be $49.57 \%$ for both sources which is high compared to the completeness of drug poisoning surveillance (17.26\%) [23].

Improvement of the completeness of poisoning surveillance system is needed to estimate the true incidence of poisoning in Morocco.

\section{References}

1. M. Helms, P. Vastrup, P. Gerner-Smidt, K. Mølbak, BMJ, 326(7385), 357 (2003)

2. R.E. Hoffman, J. Greenblatt, B.T. Matyas, D.J. Sharp, E. Esteban, K. Hodge, A. Liang, Emerg. Infect. Dis., 11, 11-16 (2005)

3. M.D. Fleury, J. Stratton, C. Tinga, D.F. Charron, J. Aramini, Can. J. Public Health, 99, 489-493 (2008)

4. Centers for Disease Control and Prevention, Estimates of Foodborne Illness in the United States, https://www.cdc.gov/foodborneburden/2011foodborne-estimates.html (2011)

5. G. Wu, Q. Yuan, L. Wang, J. Zhao, Z. Chu, M. Zhuang, Y. Zhang, K. Wang, P. Xiao, Y. Liu, Z. Du, Medicine (Baltimore), 97 (45), e13142 (2018)

6. R. Hmimou, N. Rhalem, R. Aghandous, S. Benlarabi, H. Chaoui, I. Semlali, M. Badri, R. Soulaymani-Bencheikh, Toxicologie Maroc, 31 (4), 3-7 (2016)

7. World Health Organization. Food Safety, in https://www.who.int/news-room/factsheets/detail/food-safety (2011)

8. R.M. Cormack, Oceanogr. Mar. Biol. Ann. Rev., 6, 455-506 (1968)

9. Z.E. Schnabel, Am. Math. Mon., 45, 348-350 (1938)

10. K.M. Wolter, Science, 253 (5015), 12-15 (1991)

11. International Working Group for Disease Monitoring and Forecasting, Am. J. Epidemiol., 142 (10), 1047-1058 (1995)

12. R. Van Hest, Capture-recapture Methods in Surveillance of Tuberculosis and Other Infectious Diseases (Thesis, Erasmus University Medical Center Rotterdam, 2007)

13. C. Campèse, D. Che, Evaluation quantitative du système de surveillance des légionelloses en France en 2010 (Ed. Saint-Maurice : Institut de Veille Sanitaire, 40p., 2012)

14. E.B. Hook, R.R. Regal, Epidemiol. Rev., 17 (2), 243-264 (1995) 
15. A. Gallay, A. Nardone, V. Vaillant, J.C. Desenclos, Rev. Epidemiol. Sante Publique, 50 (2), 219-232 (2002)

16. C.C. Sekar, W.E. Deming, Amer. Stat. Ass. J., 44, 100-115 (1949)

17. D.G. Chapman, Some properties of the hypergeometric distribution with applications to zoological sample censuses (Ed. Berkeley, University of California Press, 131-159p., 1951).

18. G.A.F. Seber, Biometrics, 26 (1), 13-22 (1970)

19. T.D. Mastro, D. Kitayaporn, B.G. Weniger, S. Vanichseni, V. Laosunthorn, T. Uneklabh, C. Uneklabh, K. Choopanya, K. Limpakarnjanarat, Am. J. Public Health, 84 (7), 1094-1099 (1994)

20. G.A. Seber, J.T. Huakau, D. Simmons, Biometrics, 56 (4), 1227-1232 (2000)

21. B. Hubert, J.C. Desenclos, Rev. Epidemiol. Sante Publique, 41 (3), 241-249 (1993)

22. A. Perrocheau, D. Levy-Bruhl, BEH, 51, $227-$ $229(2000)$

23. S. Hmimou, H. Hami, R. Hmimou, A. Soulaymani, A. Mokhtari, R. SoulaymaniBencheikh, Eur. Sci. J., 14 (12), 78-88 (2018) 